\title{
MODEL COMPLETE THEORIES WITH A DISTINGUISHED SUBSTRUCTURE
}

\author{
ALAN H. MEKLER ${ }^{1}$ \\ ABSTRACT. The properties of a structure of "infinite dimension" over a \\ substructure are investigated.
}

0. Introduction. In [6] Tarski asked about the decidability of the theory of a field with a distinguished subfield. Robinson [4] showed that the theory of a real closed field densely embedded in another real closed field is decidable. In the case where the larger field is algebraically closed, Keisler [2] has shown how the theory depends on the theory of the smaller field.

Suppose $F_{1} \subseteq K_{1}, F_{2} \subseteq K_{2},\left[K_{1}: F_{1}\right]=\left[K_{2}: F_{2}\right]=\infty, F_{1} \equiv F_{2}$ and $K_{1}$ and $K_{2}$ are algebraically closed. Keisler showed that $\left(K_{1}, F_{1}\right) \equiv\left(K_{2}, F_{2}\right)$. In fact he showed the conclusion holds if we use any elementary equivalent expansions of $F_{1}$ and $F_{2}$. MacIntyre [3] has given a generalization of Robinson's results by finding a generalization of "dense". He was also able to generalize Keisler's result in the case where the subfield is algebraically closed. We will generalize Keisler's results by generalizing "the dimension of $A$ over $B$ is infinite".

1. The general theory. In order to isolate the property we are looking for we will define what it means for a theory to be an enriched model completion. In the following, $L$ is a language, $U$ a unary predicate not in $L$, and, if $F \in L$, $F^{U}$ is the relativization of $F$ to $U$. If $K \subseteq L, K^{U}=\left\{F^{U} \mid F \in K\right\}$. Suppose $T \subseteq L$ and $S \subseteq L(U) . S$ is an enriched model completion of $T$ if: (i) $S \cap L$ is the model completion of $T$, (ii) $S \supseteq T^{U}$, (iii) for all $A \vDash T, S$ has a model where the interpretation of $U$ is $A$, and (iv) for all $A=T, S \cup$ $\operatorname{Th}\left(\langle A, a\rangle_{a \in A}\right)^{U}$ is complete.

Note that there is not, in general, a unique enriched model completion (see Example 1). Also, Keisler's results establish that the theory of algebraically closed fields of infinite dimension over a subfield is an enriched model completion of the theory of fields.

The first theorem is an analogue of elimination of quantifiers. This improves a result of Jarden [1].

Received by the editors April 14, 1978.

AMS (MOS) subject classifications (1970). Primary 02H05, $02 \mathrm{H} 15$.

'Research partially supported by a grant from the National Research Council of Canada (Grant \#3775). 
THEOREM 1. Assume $S$ is an enriched model completion of $T$. For any formula $F\left(x_{0}, \ldots, x_{n}\right) \in L(U)$ there exists $G\left(x_{0}, \ldots, x_{n}\right) \in L$ such that

$$
S \vdash\left(U\left(x_{0}\right) \wedge \cdots \wedge U\left(x_{n}\right)\right) \rightarrow\left(F\left(x_{0}, \ldots, x_{n}\right) \leftrightarrow G^{U}\left(x_{00} \ldots, x_{n}\right)\right) .
$$

Proof. Let

$$
\begin{aligned}
& \Sigma=\left\{G\left(x_{0}, \ldots, x_{n}\right) \in L \mid S \vdash G^{U}\left(x_{0}, \ldots, x_{n}\right)\right. \\
&\left.\rightarrow\left(F\left(x_{0}, \ldots, x_{n}\right) \wedge U\left(x_{0}\right) \wedge \cdots \wedge U\left(x_{n}\right)\right)\right\} .
\end{aligned}
$$

We wish to show there exists $G\left(x_{0}, \ldots, x_{n}\right)$ such that

$$
S \vdash\left(F\left(x_{0}, \ldots, x_{n}\right) \wedge U\left(x_{0}\right) \wedge \cdots \wedge U\left(x_{n}\right)\right) \rightarrow G^{U}\left(x_{0}, \ldots, x_{n}\right) .
$$

If not, then

$$
\begin{aligned}
R= & S \cup\left\{F\left(c_{0}, \ldots, c_{n}\right) \wedge U\left(c_{0}\right) \wedge \cdots \wedge U\left(c_{n}\right)\right\} \\
\cup & \left.\cup \neg G^{U}\left(c_{0}, \ldots, c_{n}\right) \mid G\left(c_{0}, \ldots, c_{n}\right) \in \Sigma\right\}
\end{aligned}
$$

is consistent where $c_{0}, \ldots, c_{n}$ are constants outside $L$. Let $(A, C) \vDash R$. Since $S$ is an enriched model completion, $S \cup \operatorname{Th}\left(\langle C, c\rangle_{c \in C}\right)^{U}$ is complete. So for some $H\left(c_{0}, \ldots, c_{n}, b_{0}, \ldots, b_{t}\right)$, with $b_{0}, \ldots, b_{t}$ constants outside

$$
L\left(c_{0}, \ldots, c_{n}\right)(A, C) \vDash H^{U}\left(c_{0}, \ldots, c_{n}, b_{0}, \ldots, b_{t}\right)
$$

and

$S \vdash H^{U}\left(c_{0}, \ldots, c_{n}, b_{0}, \ldots, b_{t}\right) \rightarrow\left(F\left(c_{0}, \ldots, c_{n}\right) \wedge U\left(c_{0}\right) \wedge \cdots \wedge U\left(c_{n}\right)\right)$.

Hence

$$
\exists y_{0}, \ldots, y_{t} H\left(x_{0}, \ldots, x_{n}, y_{0}, \ldots, y_{t}\right) \in \Sigma \text {, }
$$

and

a contradiction.

$$
(A, C) \vDash\left(\exists y_{0}, \ldots, y_{t} H\left(c_{0}, \ldots, c_{n}, y_{0}, \ldots, y_{t}\right)\right)^{U},
$$

This theorem justifies the statement that no new first order information is gained about a field by considering it as embedded in its algebraic closure.

We now give our generalization of $[K: F]=\infty$.

Definition. Suppose $B$ is a substructure of $A$ then $\operatorname{dim}(A: B)=\infty$ if $(A, B) \vDash D I M$ where

$$
\begin{aligned}
\operatorname{DIM}=\left\{\exists x_{0}, \ldots, x_{n} \forall y_{0}, \ldots, y_{t}\left(\bigwedge_{i \neq j} x_{i} \neq x_{j} \wedge\left(\bigwedge_{i<t} U\left(y_{i}\right)\right)\right)\right. \\
\rightarrow \bigwedge_{F \in \Sigma}\left(\bigwedge _ { \sigma : k + 1 \stackrel { 1 - 1 } { \rightarrow } n } \left(F\left(x_{0}, \ldots, x_{k}, y_{0}, \ldots, y_{t}\right)\right.\right. \\
\left.\left.\left.\leftrightarrow F\left(x_{\sigma(0)}, \ldots, x_{\sigma(k)}, y_{0}, \ldots, y_{t}\right)\right)\right)\right)
\end{aligned}
$$

$\mid \Sigma$ a finite set of quantifier free formulae in $L, n, k, t<\omega\}$. 
This says for every finite set $\Sigma$ of quantifier-free formulae, and natural number $n, A$ has $n$ elements which are indiscernible over $B$, with respect to $\Sigma$. Although this definition does not quite capture $[K: F]=\infty$, it has some nice properties:

(a) for any theory $T,\{(A, B) \mid(A, B) \vDash T$ and $\operatorname{dim}(A, B)=\infty\}$ is an $E C_{\Delta}$;

(b) when $A, B$ are fields if $\operatorname{dim}(A, B)=\infty$ then $[A: B]=\infty$. If $A$ is algebraically closed the converse is true;

(c) if $C \supseteq A \supseteq B$ and $\operatorname{dim}(A, B)=\infty$ then $\operatorname{dim}(C, B)=\infty$. If $C \supseteq A \supseteq$ $B$ and $\operatorname{dim}(C, A)=\infty$ then $\operatorname{dim}(C, B)=\infty$.

THEOREM 2. Suppose $T^{\prime}$, a countable theory, is the model completion of a universal theory $T$, and for all countable $A=T, T^{\prime} \cup \operatorname{Diag}(A)$ is $\omega_{1}$-categorical. Then $S=T^{\prime} \cup T^{U} \cup \mathrm{DIM}$ is an enriched model completion of $T$.

Proof. Clauses (i) and (ii) of the definition of an enriched model completion are satisfied. To prove properties (iii) and (iv) we will want to use facts about categoricity. For convenience our references will be to Sacks [5].

Suppose $A \vDash T$ and $|A|=\omega$. By [5, 34.1, 35.1], $T^{\prime} \cup \operatorname{Diag}(A)$ has a model $B$ with infinitely many indiscernibles. So $(B, A) \vDash S$. In general if $A \vDash T, S \cup$ $\operatorname{Diag}(A)^{U}$ is consistent. By compactness there is $\left(B, A^{\prime}\right) \vDash S$ with $A^{\prime} \supseteq A$. So by observation (c) above $(B, A) \vDash S$. This leaves. (iv) to verify.

Suppose $(B, A),(D, C) \vDash S$, and for some expansions $A^{*}, C^{*}$ of $A$ and $C$, $A^{*} \equiv C^{*}$. By passing to elementary extensions we can assume $\left(B, A^{*}\right)$ and $\left(D, C^{*}\right)$ are saturated of cardinality $\kappa>\omega_{1}$. Note that assuming the existence of saturated elementary extensions is innocuous (cf. [5, p. 81]). An isomorphism $f: A^{*} \rightarrow C^{*}$ exists since $A^{*}$ and $C^{*}$ are elementary equivalent, saturated structures at the same cardinality. We will show $f$ can be extended to an isomorphism $g: B \rightarrow D$. We need the following.

Clam. There exists $E \subseteq B\left(E^{\prime} \subseteq D\right)$ such that $|E|\left(\left|E^{\prime}\right|\right)=\kappa$ and the elements of $E\left(E^{\prime}\right)$ are indiscernible over $A(C)$ in the language $L$.

Proof of Claim. Consider the countable type obtained by dropping the existential quantifiers from the sentences of DIM. Since $(B, A) \vDash D I M$, this type is finitely satisfiable, and so by saturation is satisfied. A second appeal to saturation gives $E \subset B,|E|=\kappa$, a set of indiscernibles over $A$. Similarly $E^{\prime} \subseteq D,\left|E^{\prime}\right|=\kappa, E^{\prime}$ a set of indiscernibles at $C$ exists. Note we are using the fact that $T^{\prime}$ admits elimination of quantifiers [5, 13.1].

In his proof Keisler uses algebraically independent elements to form two transcendence bases of equal cardinality to extend the isomorphism. We shall use facts about categoricity to follow the same strategy.

Choose a countable substructure $A^{\prime}$ of $A$. So $T^{\prime} \cup \operatorname{Diag}\left(A^{\prime}\right)$ is $\omega_{1}$-categorical. First take prime model extensions of $A$ and $C$ to models of $T^{\prime} \cup$ $\operatorname{Diag}\left(A^{\prime}\right)$. By $\left[5,36.2\right.$ and 38.1], we can choose $a_{0}, \ldots, a_{n-1}$ and $c_{0}, \ldots, c_{n-1}$ such that the isomorphism $f$ can be extended to $f_{0}: A\left(a_{0}, \ldots, a_{n-1}\right) \rightarrow$ $C\left(c_{0}, \ldots, c_{n-1}\right), f_{0}\left(a_{i}\right)=c_{i}$, and there exists $p \in S_{1}\left(T^{\prime} \cup\right.$ $\left.\operatorname{Diag}\left(A^{\prime}\left(a_{0}, \ldots, a_{n-1}\right)\right)\right)$ which is a minimal generator. By $[5,35.8]$ there exist 
$E_{0} \subseteq E\left(E_{0}^{\prime} \subseteq E^{\prime}\right)$ such that $\left|E-E_{0}\right|<\omega$ and $E_{0}\left(E_{0}^{\prime}\right)$ is indiscernible over $A\left(a_{0}, \ldots, a_{n-1}\right)\left(C\left(c_{0}, \ldots, c_{n-1}\right)\right)$.

Choose $X$ a $p$-basis for $B$. Let $\left\{x_{\alpha} \mid \alpha<\kappa\right\}$ be an enumeration of $X$. Define $X^{\prime} \subset X$ inductively as follows. Let $X_{0}=0$. Let $X_{\alpha+1}=X_{\alpha}$ if $x_{\alpha}$ is algebraic over $A\left(a_{0}, \ldots, a_{n-1}\right)\left(X_{\alpha}\right), X_{\alpha+1}=X_{\alpha} \cup\left\{x_{\alpha}\right\}$ otherwise. If $\alpha$ is a limit ordinal let $X_{\alpha}=\cup_{v<\alpha} X_{v}$. Let $X^{\prime}=\cup_{\alpha<\kappa} X_{\alpha}$. Note $B$ is prime over $A\left(a_{0}, \ldots, a_{n-1}\right)\left(X^{\prime}\right)$, since $X$ is algebraic over $A\left(a_{0}, \ldots, a_{n-1}\right)\left(X^{\prime}\right)$. Further $\left|X^{\prime}\right|=\kappa$. If not, then repeated applications of $[5,35.8]$ would give $E_{1} \subseteq E$, $\left|E_{1}\right|=\kappa$ and $E_{1}$ a set of indiscernibles over $A\left(a_{0}, \ldots, a_{n-1}\right)\left(X^{\prime}\right)$. But by [5, 35.9] and the fact that $B$ is prime over $A\left(a_{0}, \ldots, a_{n-1}\right)\left(X^{\prime}\right)$ every set of indiscernibles in $B$ over $A\left(a_{0}, \ldots, a_{n-1}\right)\left(X^{\prime}\right)$ is countable. Note as well that each $x_{\alpha} \in X^{\prime}$ satisfies the unique pre-image of $p$ of rank and degree 1 in $S_{1}\left(A\left(a_{0}, \ldots, a_{n-1}\right)\left(X_{\alpha}\right)\right)$. Repeating this construction we can get $Y^{\prime}$ with the same properties relative to $C\left(c_{0}, \ldots, c_{n-1}\right)$. So we can extend $f_{0}$ to $f_{1}$ : $A\left(a_{0}, \ldots, a_{n-1}\right)\left(X^{\prime}\right) \rightarrow C\left(c_{0}, \ldots, c_{n-1}\right)\left(Y^{\prime}\right)$. Finally since $B, D$ are prime extensions of the above structures by $[5,36.2]$ there exists $g \supseteq f_{1}, g$ an isomorphism from $B$ to $D$.

The above proof gives a full generalization of Keisler's theorem.

Corollary. Let $T, T^{\prime}$ be as above. Suppose $A \subseteq B \vDash T^{\prime}, C \subseteq D \vDash T^{\prime}$ and $(B, A)(D, C) \vDash D I M$. If for some expansions $A^{*}$ and $C^{*}$ of $A$ and $C, A^{*} \equiv C^{*}$ then $\left(B, A^{*}\right) \equiv\left(D, C^{*}\right)$.

2. Applications and examples. In this section we wish to investigate some of the aspects of enriched model completions and dimension. The first question we deal with is "how many enriched model completions can a theory have?".

EXAMPLE 1. There are theories satisfying the hypotheses of Theorem 2 with $1,2^{n}, \omega$, and $2^{\omega}$ enriched model completions. The theory of fields has only one enriched model completion. This can be proved by appealing to the fact that if $[K: F]<\infty$ and $K$ is algebraically closed then $F=K$ or $F$ is real closed.

The theory of vector spaces over $\mathbf{Q}$ has two enriched model completions. The second enriched model completion is axiomatized by $\forall x U(x)$. Here a defect in our definition of DIM $=\infty$ should be pointed out. If $V \supseteq W$, $V \neq W$, then $(V, W) \vDash$ DIM even though $V$ may be finite dimensional over $W$. If $L$ is a language with countably many constant symbols $c_{n}$ and $T$ is the theory which says they are all different then $T$ has $\omega$ enriched model completions.

Finally let $L$ be as above and let $T$ be the empty theory, then $T$ has $2^{\omega}$ enriched model completions. These can be obtained by the $2^{\omega}$ ways of completing $\left\{\left(\bigwedge_{1<k<n} a_{0} \neq a_{k}\right) \rightarrow\left({\exists^{2 n+1}}^{2}\right) \neg U(x)\right\}$. We can use a variant of the above trick to get theories with $2^{n}$ enriched model completions. Suppose $n>1$. Let $L$ be the language of $Q$ vector spaces, with two additional unary predicates $A, B$, and $n+1$ new constant symbols $\left\{a_{0}, \ldots, a_{n}\right\}$. Let $T$ be the 
theory which says $a_{1}, \ldots, a_{n}$ are all different; $a_{0}$ equals one of $a_{1}, \ldots, a_{n}$; the interpretation of $A$ is $\left\{a_{1}, \ldots, a_{n}\right\} ; B$ is a $\mathbf{Q}$ vector space; the operations act trivially on $A$.

EXAMPLE 2. We now wish to show that Theorem 2 cannot be easily improved. We shall give an example of a universal theory $T$ with a model completion $T^{\prime}$, such that for every $A \vDash T, T^{\prime} \cup \operatorname{Diag}(A)$ is $\omega$-stable. This theory has no enriched model completion. The language $L$ has two unary predicate symbols $A, B$, a unary function symbol $f$, constant symbols $c_{n}$, $n<\omega$, and $d$. The models of $T$ will consist of two disjoint sets $A$ and $B$ with $c_{n} \in B$ and $d \in A$. The function $f$ acts trivially on $A$, and maps $B$ to $A$. The images of the $c_{n}$ are all different and if $x=f\left(c_{n}\right), x$ has at most $n+2$ pre-images. The model completion $T^{\prime}$ is obtained by requiring that each element of $A$ has as many pre-images as possible.

We will omit giving $T$ and $T^{\prime}$ explicitly, and proving that they have the specified properties. If $M \vDash T$, we can show its theory is completely determined by which sentences of the following form it satisfies:

(i) $\left\{f\left(c_{n}\right)=d \mid n<\omega\right\}$,

(ii) $\left\{\forall x\left(\left(f\left(c_{n}\right)=x\right) \rightarrow \exists^{k} y(f(y)=x)\right) \mid h, k<\omega, k>0\right\}$,

(iii) $\left\{\exists^{k} x \exists^{\prime} y(f(y)=x) \mid 0<k, t<\omega\right\}$.

This can be shown by characterizing the countable saturated models of $T$. Now, assume that $T$ has an enriched model completion $S$. Choose $M \vDash T$ such that $d$ has infinitely many pre-images. Suppose

$$
S \cup \operatorname{Th}\left(\langle M, m\rangle_{m \in M}\right)^{U} \vdash \exists x(f(x)=d \wedge \neg U(x)) .
$$

Then there is a finite set $R$ of sentences of the above forms or their negation such that $M \vDash R$ and $S \cup R^{U} \vdash \exists x(f(x)=d \wedge \neg U(x))$. Choose $m$ greater than any subscript or multiplicity mentioned in $R$. We can now find $N \vDash T \cup$ $R$ such that $f\left(c_{m}\right)=d$ and $d$ has $m+2$ pre-images. So $S \cup \operatorname{Th}(N)^{U}$ is inconsistent, a contradiction. A similar argument handles the case

$$
S \cup \operatorname{Th}\left(\langle M, m\rangle_{m \in M}\right)^{U_{\vdash}} \neg \exists x(f(x)=d \wedge \neg U(x)) .
$$

So $T$ has no enriched model completion.

EXAMPLE 3. Although Example 2 eliminates many possible general extensions of Theorem 2, there are theories with an enriched model completion. The theory of abelian groups has an enriched model completion. Suppose $A \subseteq B$ are abelian groups. For a prime $p$, define "DIM $p=\infty$ " if the $\operatorname{dim}_{\mathbf{z} / p \mathbf{z}}(B[p] / A[p])=\infty$, where $B[p](A[p])$ are the elements of order $p$. Note that "DIM $p=\infty$ " can be expressed by a set of first order sentences. Let $T$ be the theory of abelian groups, $T^{\prime}$ the theory of divisible abelian groups. Then $S=T^{\prime} \cup T^{U} \cup\{$ \{DIM $p=\infty " \mid p$ a prime $\}$ is an enriched model completion of $T$. To see this we can characterize saturated models of $S$. If $(A, B)$ is saturated of cardinality $\kappa, A$ is the sum of the divisible hull of $B$ with $\kappa$ copies of each $Z\left(p^{\infty}\right)$, and $Q$. 
EXAMple 4. Finally we wish to exploit our definition of "DIM $=\infty$ " to show that the result "if $[K: F] \neq \infty$ and $K$ is algebraically closed then $F$ is real closed or algebraically closed" does not seem to follow solely from model theoretic reasons. We will give theories $T, T^{\prime}$ which closely resemble that of fields and algebraically closed fields. In addition, $T$ will have $2^{\omega}$ nonelementary equivalent models $A_{i}, i \in I$, such that for each $A_{i}$ there exists $B_{i} \vDash T^{\prime}$ and $(B, A) \vDash \neg$ DIM.

Let $L$ be the language of fields augmented by two unary predicates, and a unary function symbol. Models of $T$ will be a disjoint union of the two distinguished subsets, the function will embed the first set in the second. The second set will be a field, outside their intended domain all functions will act trivially. We can think of our model as a field with certain of its elements coloured. To get $T^{\prime}$ we demand the second set be an algebraically closed field and the function a bijection. To get our $A_{i}$, take $\mathbf{R}$ with different colourings.

\section{REFERENCES}

1. M. Jarden, Algebraically closed fields with distinguished subfields, Arch. Math. (Brno) 27 (1976), 502-505.

2. H. J. Keisler, Complete theories of algebraically closed fields with distinguished subfields, Michigan Math. J. 11 (1964), 71-81.

3. A. Macintyre, Dense embeddings. I: a theorem of Robinson in a general setting, in model theory and algebra, A Memorial Tribute to Abraham Robinson, Lecture Notes in Math., vol. 498, Springer-Verlag, Berlin nnd New York, 1975 pp. 200-219.

4. A. Robinson, Solution to a problem of Tarski, Fund. Math. 47 (1959), 179-204.

5. G. Sacks, Saturated model theory, Benjamin, New York, 1972.

6. A Tarski and J. C. C. McKinsey, A decision method for elementary algebra and geometry, Rand Corporation, Santa Monica, 1948.

Department of Mathematics, Carleton University, Ottawa, Ontario, Canada

Current address: Department of Mathematics, University of Toronto, Toronto, Ontario, Canada 